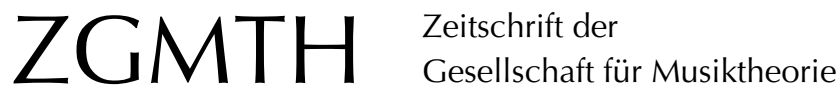

Lehner, Michael (2010): »So fängt nur Chopin an, ... so schließt nur er«. Initial- und Finalgestaltung in Chopins Mazurken. ZGMTH 7/3, 345-360.

https://doi.org/10.31751/600

(C) 2010 Michael Lehner

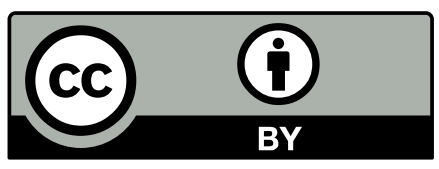

Dieser Text erscheint im Open Access und ist lizenziert unter einer Creative Commons Namensnennung 4.0 International Lizenz.

This is an open access article licensed under a Creative Commons Attribution 4.0 International License.

veröffentlicht / first published: 22/12/2010

zuletzt geändert / last updated: 21/05/2011 


\title{
»So fängt nur Chopin an, ... so schließt nur er« Initial- und Finalgestaltung in Chopins Mazurken
}

\author{
Michael Lehner
}

Bereits Robert Schumann betonte Frédéric Chopins Hang zur individuellen Gestaltung von Anfängen und Enden. Im Sinne der Innovationsästhetik des 19. Jahrhunderts suchte Chopin in allen Gattungen nach immer neuen Möglichkeiten, Musik beginnen und enden zu lassen. Am Beispiel seiner Mazurken werden einige dieser Strategien aufgezeigt. Dabei soll die Idee von zyklischen Formen und das Prinzip der Rahmenbildung ebenso zur Sprache kommen wie die zahlreichen, ins Offene verweisenden Schlussbildungen, die ohne die romantische Vorstellung des Fragmentarischen nicht zu denken sind.

Beginn und Schluss eines Musikstückes nehmen gemeinhin einen prominenten Platz in der Erinnerung des Hörers ein. Der Beginn spielt mit Hörerwartungen, die erfüllt oder gebrochen werden können: Ein smagischer Momentı, der selbst bei genauer Kenntnis der Musik immer wieder überraschen kann. ${ }^{1}$ Auch ein Ende oszilliert als Steigerung, als Verklärung, als Zusammenfassung, als Verdichtung als Absterben oder >Ausklang، etc. zwischen Erfüllung oder Brechung der Hörerwartung. Anfang und Schluss entscheiden maßgeblich, wie formal geschlossen oder offen, wie zusammenhängend, zyklisch oder linear entwickelt wir ein Stück Musik wahrnehmen. In der Kompositionsgeschichte stoßen wir daher sowohl auf konventionalisierte Muster (im Falle des `Beginns beispielsweise auf die klassische Introduktion $`$ ), als auch auf deren kritische Reflexion. ${ }^{2}$

Im Zuge der im 19. Jahrhundert zunehmend wirkungsmächtigeren Innovationsästhetik entstanden immer ungewöhnlichere Anfangs- und Schlussgestaltungen. Die extrem ausdifferenzierte Harmonik, die Ernst Kurth nach der Mitte des Jahrhunderts in eine `Kri-

1 Nicht umsonst wird diese Spannung vor dem eigentlichen Beginn im Opern- und Konzertbetrieb auch äußerlich als Ereignis inszeniert: durch das Löschen des Lichts, das Öffnen des Vorhangs, das kurze Verharren des Dirigenten nach dem Heben des Taktstocks; vgl. hierzu auch Cone 1968, 11-32. Ebenfalls eine prominente Position nehmen erste Takte oft in Analysen ein, ohne dass sie die Funktion und Besonderheit des Anfangs für den Fortgang reflektierten. Das in dieser Hinsicht am meisten strapazierte Beispiel scheint Richard Wagners Vorspiel zu Tristan und Isolde zu sein: Zahllose Analysen lassen vergessen, dass nach den ersten Takten noch etwa vier Stunden Musik folgen.

2 Eines der bekanntesten Beispiele der Musikgeschichte ist Machauts Rondeau Ma fin est mon commencement et mon commencement ma fin; vgl. dazu Danuser 1995. Generell zum Ende als Kategorie menschlichen Denkens und Handelns vgl. Stierle/Warning 1996. 
se der romantischen Harmonik $\imath^{3}$ münden $s^{4}{ }^{4}$, hatte Folgen für die formkonstituierende Funktion der Tonalität: Neue Initial- und Finalgestaltungen - häufig im Dienste einer romantischen Ästhetik der Formentgrenzung - wurden durch Erweiterung, Verfremdung oder Aufhebung traditioneller kadenzharmonischer und -metrischer Muster möglich.

Bereits Robert Schumann erkannte die besondere Rolle Frédéric Chopins innerhalb dieser Entwicklung. In einer Mischung aus Bewunderung und Kritik konstatiert er in seiner Rezension der Sonate in b-Moll op. 35: "So fängt nur Chopin an, und so schließt nur er: mit Dissonanzen durch Dissonanzen in Dissonanzen. $\aleph^{5}$ Und in der Tat fällt am Werk Chopins ein Hang zu höchst individueller Initial- und Finalgestaltungen auf. Vor allem die außergewöhnlichen Anfänge der großformalen Werke wie der Balladen und Scherzi wären hier anzuführen, aber auch die Préludes, die Schumann als miniaturhafte Etüdenanfänge charakterisiert. ${ }^{6}$

Im Folgenden sollen anhand der frühen Mazurken exemplarisch einige formale Strategien, mit denen Chopin temporale Extreme gestaltet, einander gegenübergestellt, sowie bezüglich der Gesamtgestaltung des jeweiligen Stückes auf ihre Funktion hin untersucht werden. Dabei erscheinen die Mazurken hinsichtlich Gattungsnorm und tänzerischer Provenienz dafür prädestiniert, formal viel strenger als andere Chopinsche Genres organisiert zu sein. Gerade aber im Schwanken zwischen Konvention und Konventionsverstoß, im Spannungsfeld zwischen Freiheit und Gebundenheit liegt ihr besonderer Reiz. ${ }^{7}$

\section{Zyklische Formen}

Chopin entwickelt bereits in seinen ersten Mazurken Formkonzepte, welche die lineare Abfolge sAnfang - Mitte - Schluss` brechen und alternative Wahrnehmungsmodi anbieten, so z. B. in der Mazurka op. 7,5, die mit insgesamt 20 Takten (davon vier Takte Einleitung) die kürzeste Chopins ist und deren tonaler Verlauf überschaubar bleibt: Der zweite Teil ab Takt 13 stellt eine Transposition des ersten Teils in der Tonart der V. Stufe dar; beide Male erklingen lediglich Tonika und Dominante. Die Schlichtheit der wirbelnden, sich immer wiederholenden Melodie im Charakter des Obere $k^{8}$ und die Orgelpunktbildung stellen die folkloristischen Wurzeln der Mazurka besonders deutlich heraus. Die viertaktig pulsierende Einleitung auf der Oktave G-g kann als Allusion von Bass- bzw. Schlagwerkbegleitung einer Musikantengruppe gehört werden. ${ }^{9}$ Dabei sind Taktart, Me-

3 Kurth 1923.

4 Gołąb konstatiert in seiner Studie über Chopins Harmonik dessen essentielle Bedeutung für das 19. Jahrhundert und kritisiert, dass eine Würdigung in Ernst Kurths Studie fehle. (1995, 9).

5 Schumann 1914, Bd. 2, 13-15.

6 Schumann ebd., Bd. 1, 418; ein Gedanke, den Anselm Gerhard (1996) aufnimmt, leider aber nicht weiterführt.

7 Die einzige deutschsprachige monographische Studie ist Schaefer 2007.

8 Vgl. Lissa 1973.

9 Einleitungen über Orgelpunkt- und Bordungestaltung sind in den Mazurken auf Grund des folkloristischen Bezugs sehr häufig. Dabei erfahren Sie vielfältige Ausprägung vom einfachsten Falle des bloßen Repetierens bis zu chromatischen und metrischen Ausgestaltungen. 

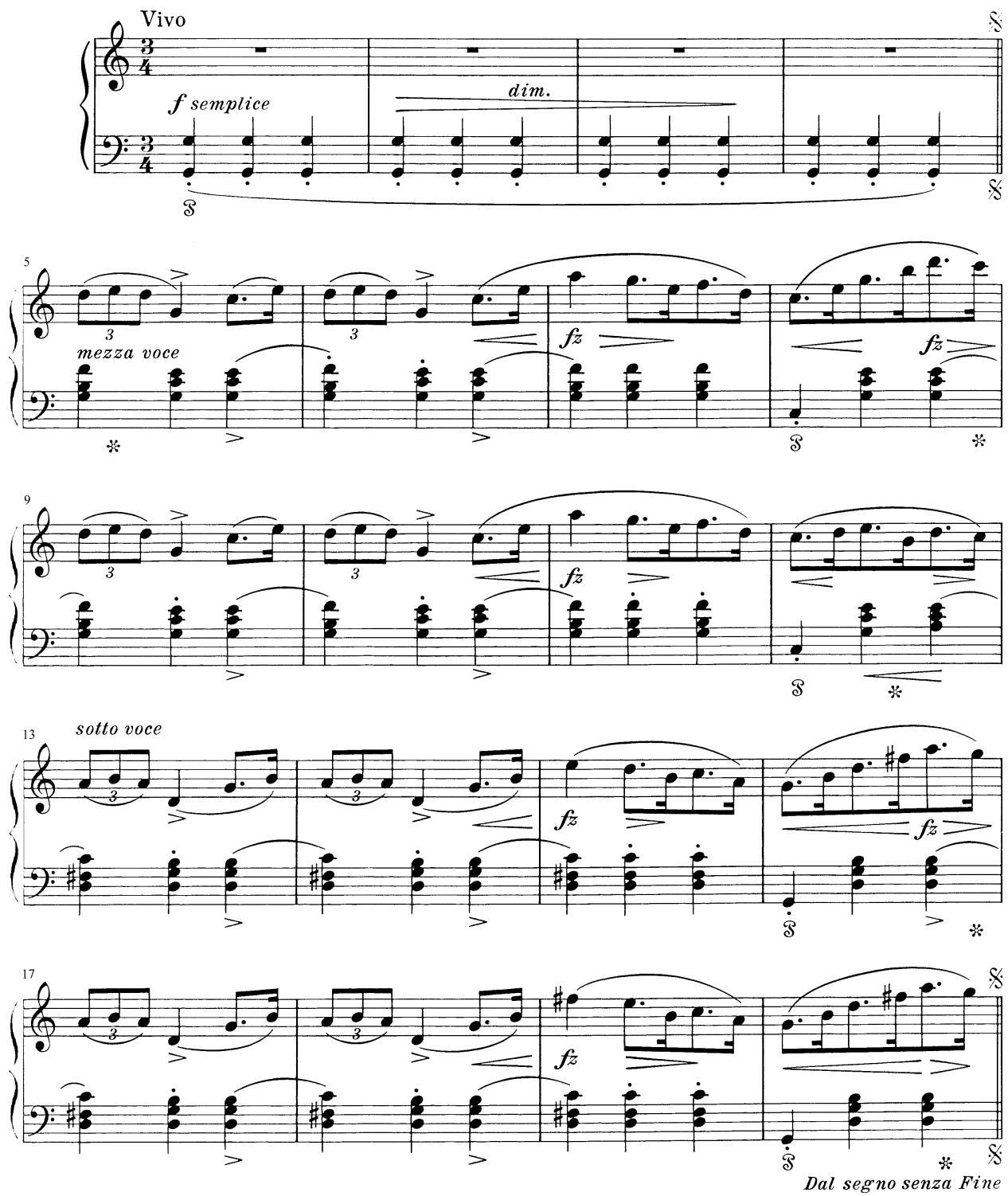

Beispiel 1: Frédéric Chopin, Mazurka op. 7,5

trum und Tonart erst mit Beginn der Melodie identifizierbar, zunächst erklingt nichts als ein regelmäßiger Puls.

Dal segno senza fine steht unter dem letzten Takt. Gemeint ist folglich ein perpetuum mobile: Sind TänzerInnen oder Musiker erschöpft, bricht der rauschhafte Tanz schlichtweg ab. Nur, nach wie vielen Wiederholungen und wo soll der Interpret das kleine Stück 
beenden? Da der G-Dur Teil zum ersten Achttakter in C-Dur eine formale Dominantspannung aufbaut, wäre ein Ende mit Takt 20 untypisch. Die dreiteilige ABA Form wird auch bei unausgeschriebenen Wiederholungen des A-Teils über die Anweisung D.C. al Fine gewährleistet, um eine tonale und formale Rundung zu erreichen. Zu erwarten wäre folglich ein Ende mit einem C-Dur Klang in Takt 12. Dies verunmöglicht Chopin jedoch: Zum einen wird die erwartete Zäsur durch die Fortsetzung des rhythmischen Patterns überspielt, zum anderen erklingt bereits auf Zählzeit 3 ein a-Moll Akkord, der eine in den 2. Teil überleitende II-V-I Folge eröffnet. Entweder also greift der Interpret in den Notentext ein, um in Takt 12 schließen zu können (wie es z. B. in der Aufnahme Arie Vardis geschieht ${ }^{10}$ ), oder er endet in Takt 20 und lässt die tonartliche Spannung zwischen beiden Teilen unaufgelöst (wie z. B. Vladimir Ashkenazy ${ }^{11}$ ). Dann entsteht ein Schluss, der sich in seiner Spannung wieder auf den Anfang bezieht; der Beginn auf dem Ton $g$ und das Ende in G-Dur bilden ein weiteres zyklisches Moment.

Bemerkenswert ist, dass dieser Tanz trotz seiner Verweigerung von Finalität das letzte Stück von op. 7 bildet, insbesondere, wenn man an die finalen Mazurken der späteren Serien denkt, die in der Mehrzahl dem Motto `Finis coronat opus` folgen (Beispiel 1).

Ein ähnlich kreisgängiger Gedanke prägt die Mazurka op. 33,1. Das Stück beginnt, »as if in the middle $\ll^{12}$, mit einer Kadenz in der Haupttonart von stark finalem Charakter. Diese wird auf der dritten Zählzeit mit einer vom Grundton skalar aufsteigenden Melodie unbegleitet weitergeführt; gis-Moll bleibt aufgrund der unmissverständlichen Setzung präsent. Die Melodie führt nach zwei plagalen Kadenzen auf den schwachen Zählzeiten der beiden Folgetakte abermals in die authentische Wendung des Anfangs. Der Nachsatz, die Wiederholung der Takte 5 bis 8, setzt, wie schon die melodische Gestalt in Takt 2, im Schlusstakt ein und verhindert so, als Auftakt für das Folgende, dass mit einer formal deutlich abschließenden Kadenz zur Ruhe gekommen wird (Beispiel 2). ${ }^{13}$

Wenn dann am Schluss der Komposition die authentische Kadenz tatsächlich in ihrer Funktion als finaler Ruhepunkt Verwendung findet, erwartet der Hörer aufgrund des bis dato unausgesetzten Konventionsverstoßes gleichwohl die erneute Fortsetzung. ${ }^{14}$ Der Interpret kann die Hörerwartung allenfalls durch ein ritardando abschwächen, das Chopin - im Gegensatz zu vielen anderen Mazurken - hier freilich nicht fordert. So sind die letzten Takte der Mazurka ein Paradox, Schluss und Nicht-Schluss gleichermaßen. Auch dieses Stück könnte sich als unendliche Form ohne Anfang und Ende im Kreise drehen (Beispiel 3).

10 Arie Vardi, Chopin: Mazurkas, Acum 1991.

11 Vladimir Ashkenazy, Chopin: Mazurkas, Decca 1995. Eine - hinsichtlich des tonalen Verlaufs ähnliche - dritte Alternative wäre denkbar: die Rückkehr in die Einleitungstakte. Hierfür entscheidet sich Artur Rubinstein, Chopin, RCA 1959-1966.

12 Rosen 1995, 419.

13 Auf gleiche Art geschieht auch die Überführung in den Mittelteil in H-Dur: Hier ermöglicht die chromatische Erniedrigung des Basstones gis zu g die harmonische Öffnung und Entwicklung.

14 Die Bedeutung von Wiederholungen und ihre Folgen für die Hörerwartung können für Chopins Mazurken gar nicht überschätzt werden. Zu Chopins Umgang mit Wiederholungspassagen vgl. Kallberg 1988 und Metzner 2004. 

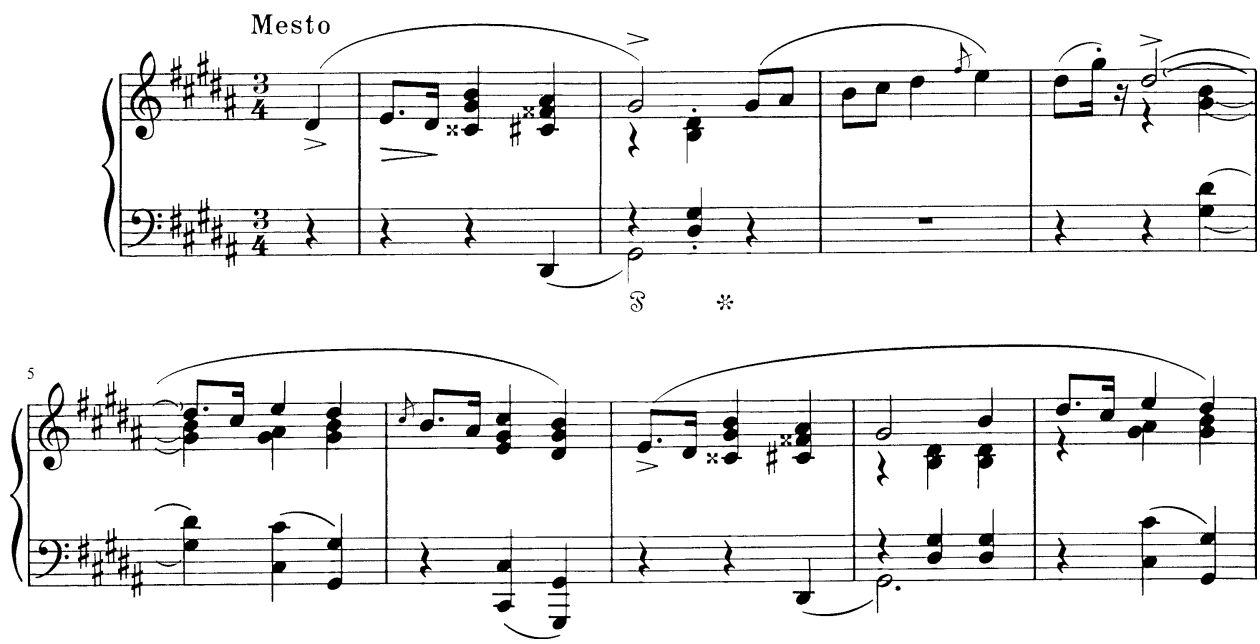

Beispiel 2: Frédéric Chopin, Mazurka op. 33,1, T. 1-9

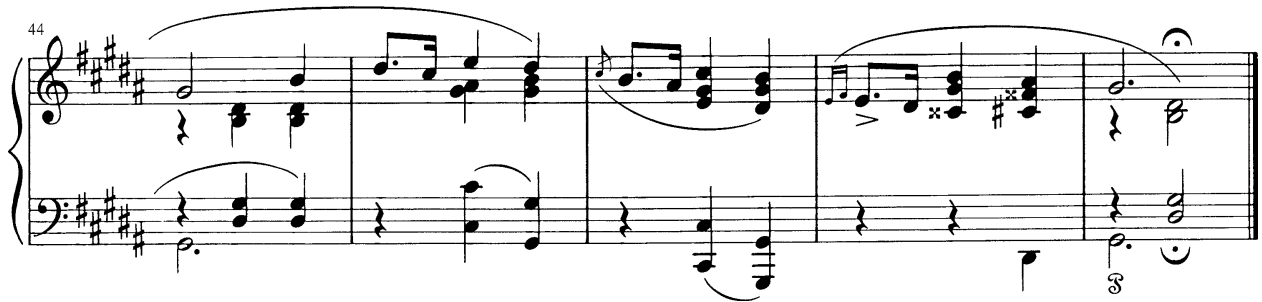

Beispiel 3: Frédéric Chopin, Mazurka op. 33,1, T. 44-48

\section{Rahmen}

Die Kadenzwendung in op. 33,1 umgibt die Form mit einem Rahmen. Dergleichen geschieht in Chopins Mazurken häufig mit Hilfe eines Borduns am Anfang und Ende der Komposition. Dadurch entsteht eine Art `Hohlformı, in die das Stück eingebettet wird. In op. 6,3 erscheint der Bordun auch inmitten der Komposition: Jeweils nach Beendigung der thematischen Achttakter wird er durch das Übergreifen der Hände wieder an die 'Oberfläche` getrieben. Dabei kommt es zu Klangflächen auf allen drei Hauptstufen: der I. Stufe (T. 1-8), der V. Stufe (T. 17-20, T. 29-32 und T. 77-80) und der IV. Stufe (T. 65-68). Auch dort, wo der Bordun nicht manifest ist, bleibt er beständig subkutan fühlbar: ein starker Anklang an den volkstümlichen Ursprung der Form. Selbst noch die vier letzten Takte (T. 87-90), die als standardisierte IV-V-I-Kadenz vom regulären Bordun abweichen, verweisen mit ihren pulsierenden Quinten e- $h$ und a-e und infolge des mit Takt 5 identischen Rhythmus' der Bassfigur auf die Einleitungstakte. ${ }^{15}$

15 Als weitere Beispiele für dieses Verfahren wären op. 56,2, op. 41,2 oder op. 24,2 zu nennen, wo der Bordun zum Akkordpendel erweitert ist. 
Einen deutlichen Rahmen hat auch op. 17,4 ${ }^{16}$ : Die einleitenden vier Takte dienen zugleich als Ausklang:
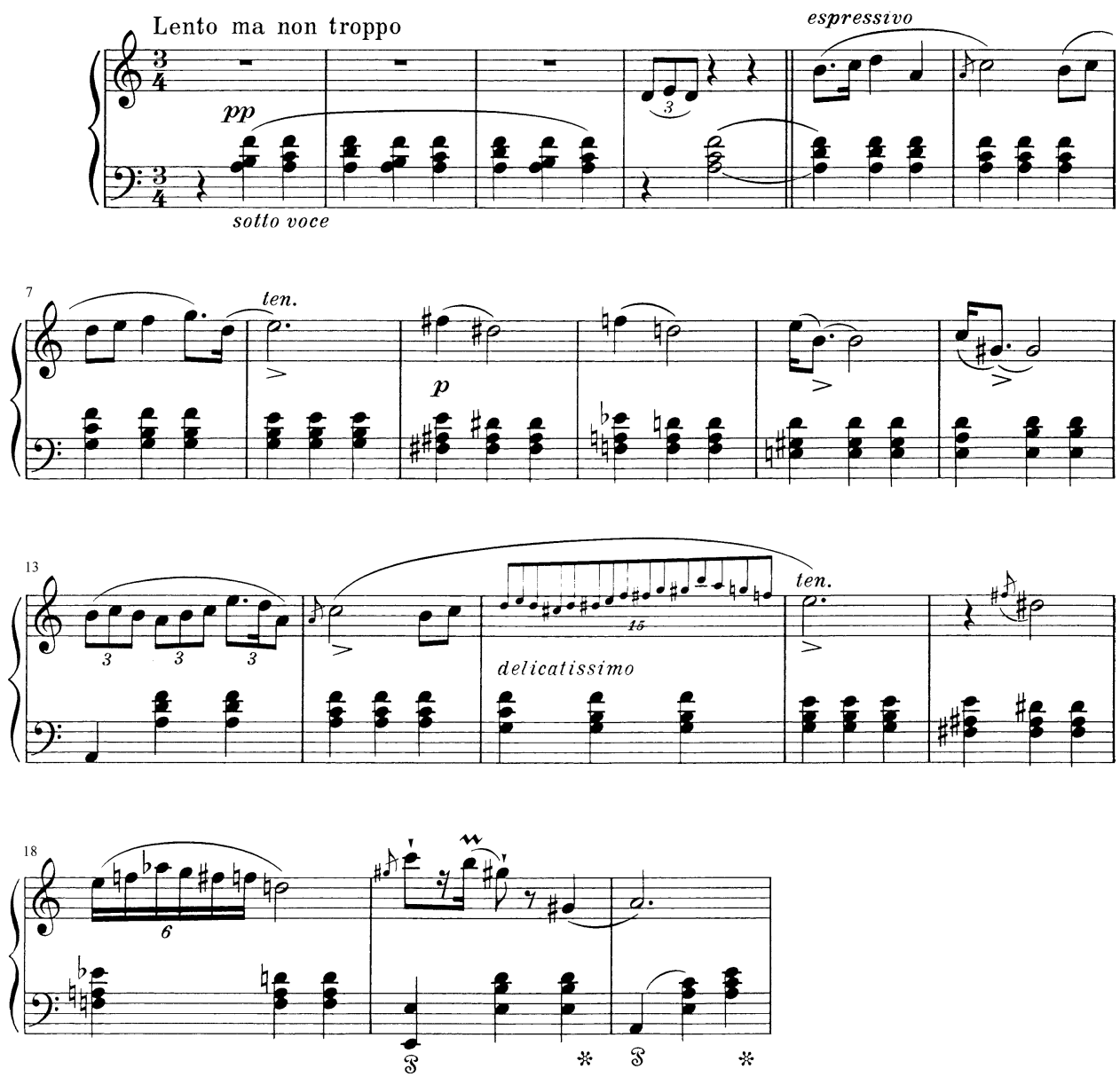

Beispiel 4: Frédéric Chopin, Mazurka op. 17,4, T. 1-20

Weder Tonart noch Taktschwerpunkte sind zu bestimmen. Inmitten einer wiederholt angeschlagenen Sexte pendelt die melodische Bewegung von $h$ über $c$ nach $d^{1}$. Der F-Dur Sextakkord, auf dem die Bewegung zur Ruhe kommt, legt als Tonart F-Dur nahe. Das wiederholt erklingende $h$ verhindert jedoch diese Lesart und lässt an eine modale Färbung denken, auf die Chopin in seinen Mazurken immer wieder zurückgreift. ${ }^{17}$ Auch

16 Zu dem Stück existieren mehrere Analysen, vgl. Borris 1963 und Chailley 1963.

17 So z. B. in op. 24,2, 41,4, etc. - Rosen betont allerdings mit Recht, dass der Begriff der >Modalität bei Chopin mit Vorsicht zu gebrauchen ist. 
eine subdominantische Tendenz im Rahmen von C-Dur oder a-Moll wäre denkbar. Mit dem Einsatz der Melodie bleibt die Rahmensexte erhalten. Erst in Takt 7 scheint endlich Klarheit bezüglich der Frage der Tonart zu bestehen: Eine Kadenz nach C-Dur bahnt sich an. Jedoch folgt die Flucht in einen weiteren Sextakkord e-Moll. Unschwer erkennbar ist nun das eigentliche Klangbildungsprinzip: ein synkopiertes Fauxbourdon-Modell, zunächst diatonisch, schließlich chromatisch absinkend.

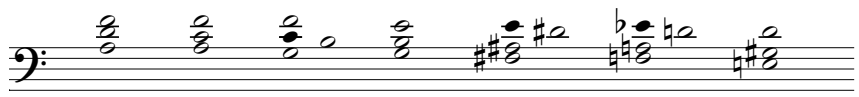

Beispiel 5: Frédéric Chopin, Mazurka op. 17,4, T. 5-11, Reduktion

Chopin erzeugt eine harmonische Mehrdeutigkeit, die sich einer funktionsharmonischen Einordnung entzieht und durch das chromatische Hinabgleiten der funktionsfreien Dominantseptakkorde - eine Vorwegnahme seiner späteren unvermittelten Mixturbildungen $^{18}$ - zur Orientierungslosigkeit führt. Eine klare Kadenz nach a-Moll in Takt 13 wird abermals durch direkte Wiederholung des vorherigen Achttakters unterbunden, so dass es zur ersten, die Tonart festigenden Kadenz erst in Takt 20 kommt.

Dabei steht diese Passage nicht isoliert in Chopins Schaffen: Wie eine Antizipation erscheinen die Takte $21 \mathrm{ff}$. der Mazurka op. 7,2. Und auch in op. 6,3 gibt es eine vergleichbare Folge (T. $57 \mathrm{ff}$.). Von den ersten Mazurken bis zu op. 68, $4^{19}$ lässt sich die chromatische Verwendung dieses Satzmodells nachweisen, obgleich das berühmteste Beispiel - zumindest in der analytischen Literatur - sicherlich das Prélude e-Moll op. 28,4 ist.
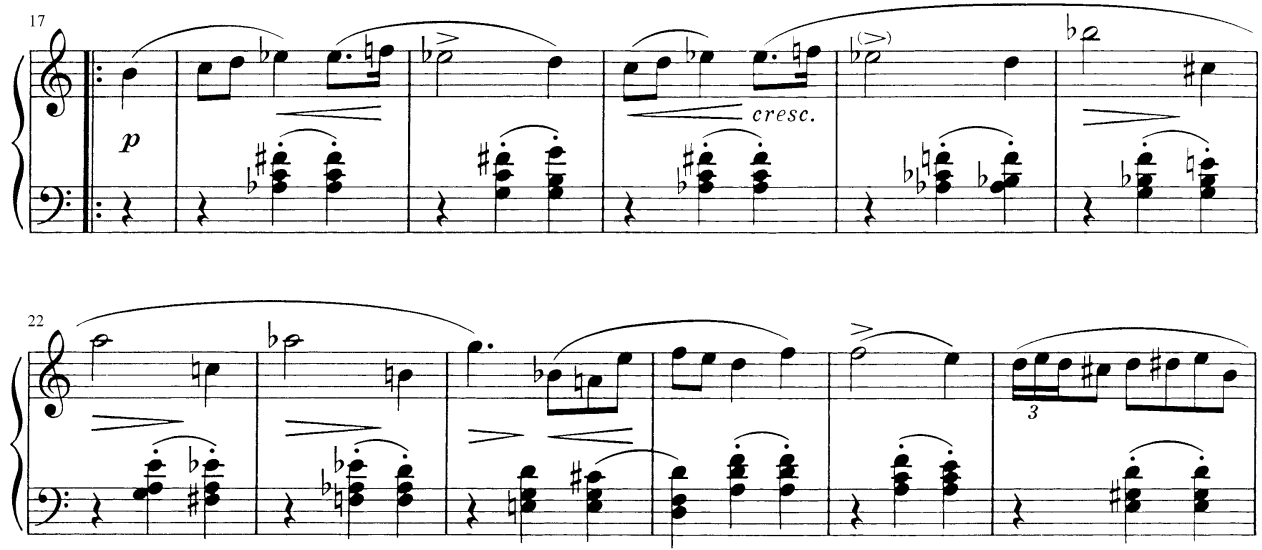

Beispiel 6: Frédéric Chopin, Mazurka op. 7,2, T. 17-27

18 Etwa in op. 30,4, T. 129-132. Auch Schumann erwähnt die auffällige Klangbildung (1914, Bd. 1, 371).

19 Vgl. Kallberg 1996 und Torkewitz 2004. 
Die ersten vier Takte von op. 17,4 übernehmen eine Doppelrolle: Einerseits bilden sie eine abgeschlossene syntaktische Gruppe und sind der Komposition mottoartig vorangestellt; ${ }^{20}$ andererseits enthalten sie die subthematische Essenz der Komposition: Der Sextakkord ist der Zentralklang des folgenden Modells, die kreisende Mittelstimme antizipiert den Anfang der Melodie $h$-c-d, der im Übergang von Takt 6 zu Takt 7 sofort wiederaufgegriffen wird und - nach Dur gewendet - im Mittelteil ab Takt 61 in Umkehrung erscheint. Und schließlich bleibt das Rahmenintervall der Melodie in den fallenden kleinen Terzen der Takte 9 und 10 prägend.

Die Wiederkehr der einleitenden vier Takte am Ende überrascht. Der ab Takt 109 einsetzende Schlussteil hat eindeutig die Funktion einer Coda und fasst als Synthese Rahmen- und Mittelteil des Stückes zusammen: Die Orgelpunktstabilität des kontrastierenden B-Teils trifft auf das chromatische Absinken des A-Teiles, dessen harmonische Instabilität durch die herausgestellten Tritoni zusätzlich gesteigert ist. Eine beständige Reduktion setzt ein: ein Ab-, schließlich Ausklingen, das nach der letzten plagalen Kadenz (T. 123f.) in Takt 128 in den Einzelton $a^{1}$ mündet. Doch überblenden den verklingenden Schlusston - diesmal perdendosi und portato - ab Takt 129 die vier Takte des Anfangs. Die funktionale Ambiguität, die das ganze Stück bestimmt hat, wirkt auch nach dessen Ende fort: Der F-Dur Sextakkord führt angesichts der Haupttonart a-Moll zu einer schwebenden Unabgeschlossenheit, während die Wiederkehr des Viertakters hingegen eine Rahmung bedeutet: "well-rounded and yet open «. ${ }^{21}$ Die vorausgehende Coda übernimmt hierbei eine entscheidende Funktion: Der klar komponierte Ausklang über dem Orgelpunkt erzeugt ein Gefühl von Finalität, das es dem Hörer verunmöglicht, die letzten Takte als möglichen Neuanfang wie in op. 33,1 zu hören. So wird der Schluss nicht in Frage, aber ins Offene gestellt.

Eine weitere Mehrdeutigkeit prägt dabei das gesamte Stück: Während der Mittelteil eindeutig eine Mazurka darstellt, lässt sich dies vom Rahmenteil nicht ebenso strikt behaupten. Zwar sind auch hier charakteristische Elemente vorhanden, insbesondere aber durch die Fiorituren und andere Verzierungen erzeugt Chopin zugleich einen nocturneartigen Charakter. So ließe sich das Stück auch als hybride Gattungskonzeption verstehen ${ }^{22}$, in der nicht nur Tonart, Taktschwerpunkt, Anfang und Ende mehrdeutig sind, sondern die Gattung selbst. Dergleichen wäre ohne die romantische Ästhetik des Fragmentarischen nicht denkbar (Beispiel 7).

\section{Große Formen}

Ab Mitte der 1830er Jahre komponiert Chopin zunehmend große Mazurken, die in formaler Gestaltung, Harmonik und Satztechnik ausgesprochen komplex sind. ${ }^{23}$ Erst mit

20 Solche exterritorialen Motti, die der Komposition vorangestellt sind, finden sich häufig in Chopins OEuvre, etwa im Nocturne cis-Moll op. 27,1.

21 Rosen 1995, 419.

22 Es gibt mehrere Beispiele für solche Gattungsmischungen: z.B die Nocturne op. 15,3 oder die Polonaise op. 44, die beide Mazurken-Elemente enthalten.

23 In den 30er Jahren sind dies die opp. 24, 30, 33, 41, in den 40er Jahren die opp. 50, 56 und 59. 

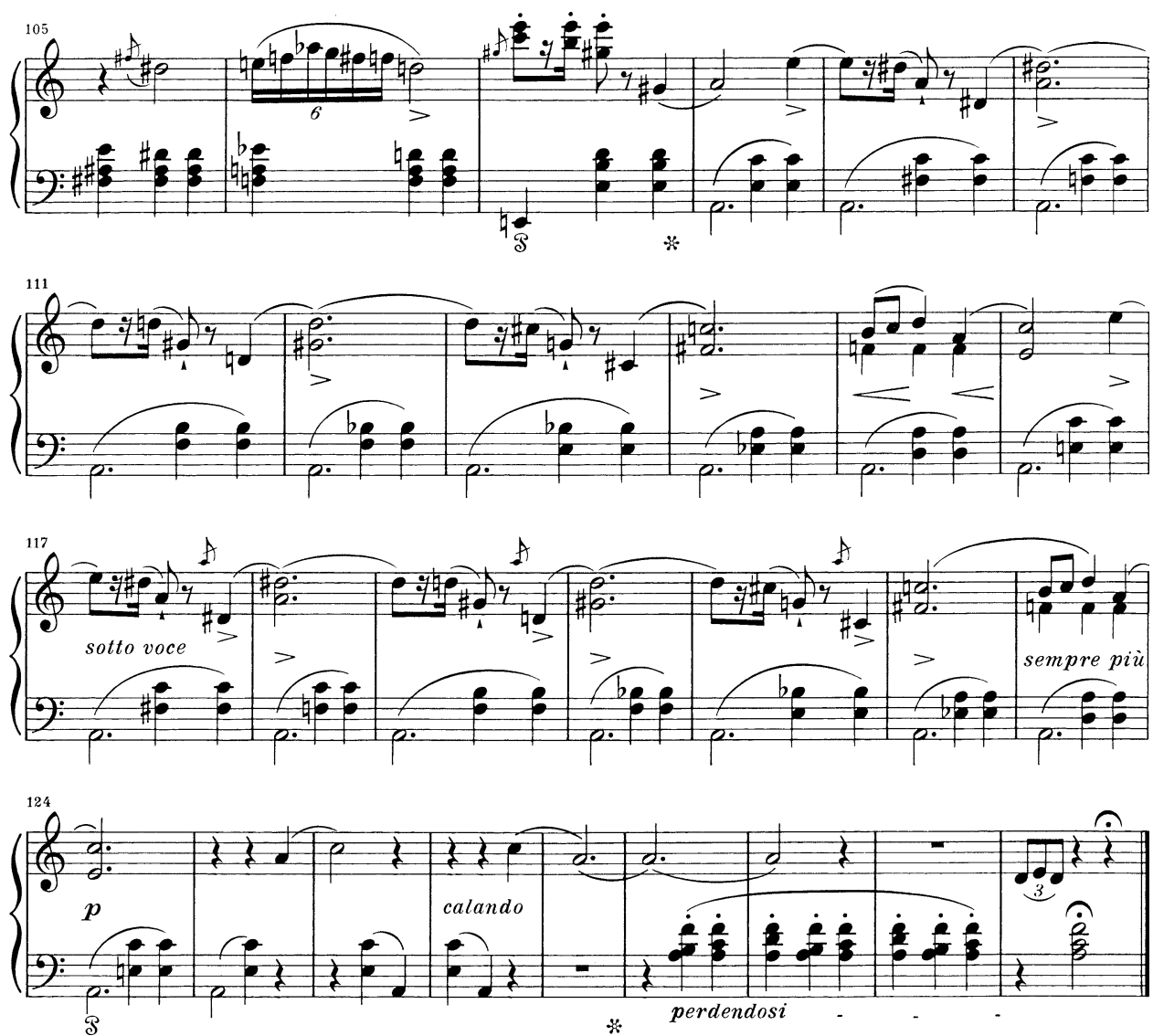

Beispiel 7: Frédéric Chopin, Mazurka op. 17,4, T. 105-132

seinem letzten Zyklus, op. 63, findet Chopin wieder zu einer schlichteren, nunmehr höchst reduzierten Schreibweise zurück. ${ }^{24}$ Bereits in op. 17,4 war diese Tendenz spürbar. Am Beispiel seiner ersten größer angelegten Mazurka, dem Finalwerk aus der Serie op. 24, sollen die Folgen dieser Entwicklung für die Anfangs- und Schlussgestaltung erörtert werden.

Chopin beginnt mit einem zweistimmigen kontrapunktischen Modell ${ }^{25}$ : Aus der Oktave $f^{1}-f^{2}$ bewegen sich zwei Stimmen chromatisch gegenläufig aufeinander zu. Eine tonale Einordnung ist zunächst unmöglich, auch nicht bei Eintritt der Begleitung zur doppelt verminderten Quinte $g i s^{1}-d e s^{2}$ der Oberstimmen (T. 5).

24 Diese Tendenz gilt auch für die nachgelassene Mazurka op. 67,4, die wie op. 63 im Jahr 1846 entstanden ist, und für die Mazurken op. 67,2, und op. 68,4.

25 Polyphone Satztechniken setzt Chopin auch in seinen großen Mazurken op. 50,3 und op. 56,3 intensiviert ein, was beide Stücke ebenfalls bereits am Anfang deutlich herausstellen. 
Moderato
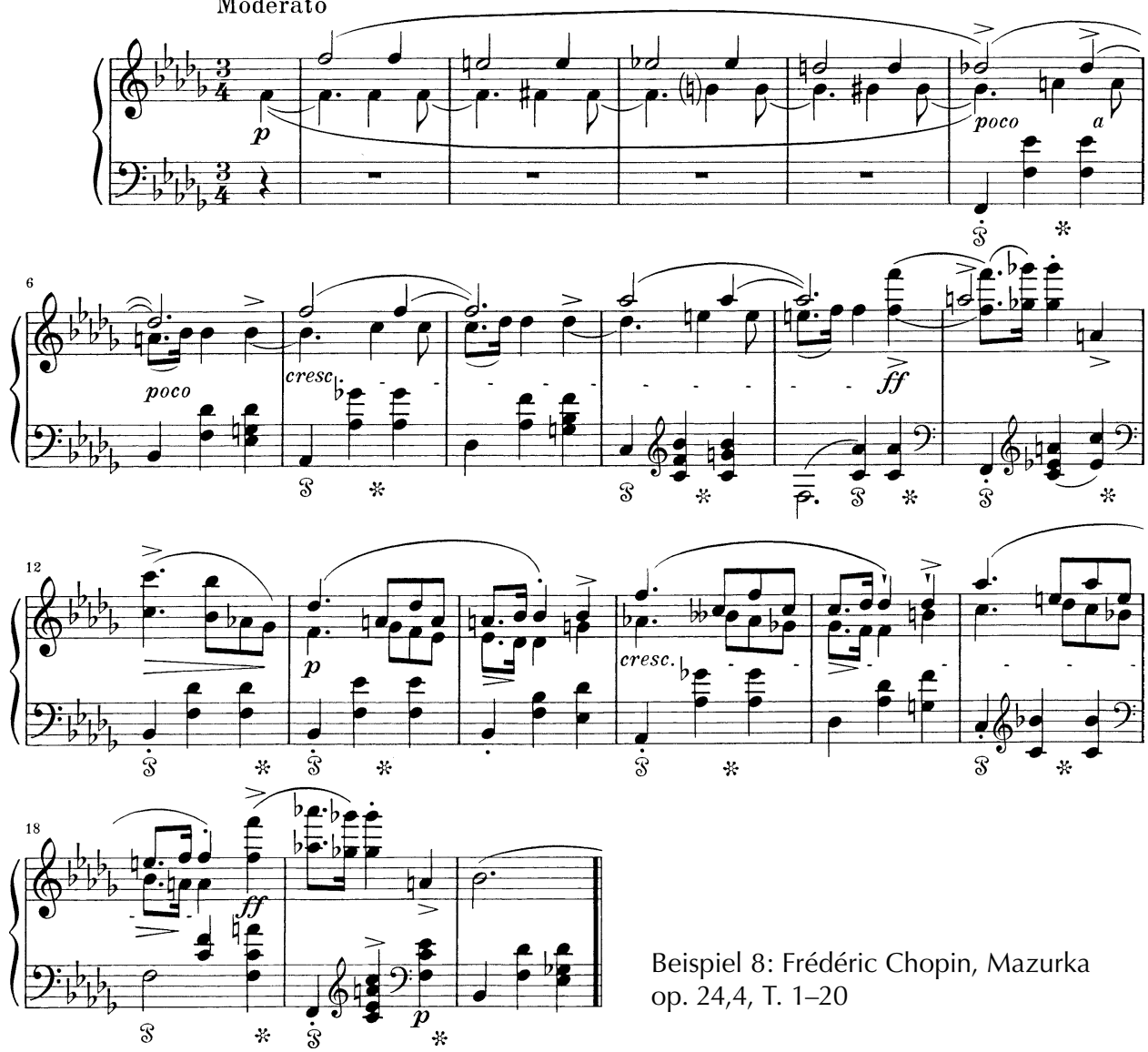

Beispiel 8: Frédéric Chopin, Mazurka op. 24,4, T. 1-20

Erst durch die Fortführung des gis ${ }^{1}$ als chromatischem Strebeton zur Terz a des charakteristischen $\mathrm{D}^{13}$ Akkordes entsteht tonale Klarheit. Die reguläre Auflösung der Dominante führt in Takt 6 in die Tonika b-Moll. Die zweistimmige Passage erschließt sich somit retrospektiv als Prolongation der Dominante. Fließend leitet sie in das eigentliche Thema und bildet mit diesem eine unauftrennbare Einheit: ein Verfahren, das die früheren Mazurken nicht kennen. Die Verbindung von Einleitung und Hauptteil zeigt sich auch auf kontrapunktischer und rhythmischer Ebene: Die Kontraktion der Oktave $f^{1}-f^{2}$ (T. 1) gelangt über die doppelt verminderte Quinte gis ${ }^{1}-d e s^{2}$ (T. 5) in die tonikale Terz $b^{1}-d e s^{2}$ (T. 6). Auch bei der Erweiterung der authentischen Kadenz zum mazurka-typischen Parallelismus-Modell ${ }^{26}$ (T. 7-10) ist das Verhalten der Oberstimmen bei den beiden folgenden Sequenzgliedern durch das `Zusammenziehen` von Quinte zu Terz gekennzeichnet. 
Das Modell der Einleitung wiederum wird ab Takt 6 um den charakteristischen MazurkaRhythmus ergänzt. ${ }^{27}$

Mit der Wiederholung des Themas ab Takt 13 kommt es zu einer Vereinfachung des Satzbildes. Deutlich wird nun, dass zuvor kein zweistimmiger Satz im strengen Sinne gemeint war, sondern ein strukturell einstimmiger Vorgang in Art des durchbrochenen Satzes in eine virtuelle Zweistimmigkeit mit ausnotierten Liegetönen transformiert wurde. Nun, da die strukturelle Einstimmigkeit in der Oberstimme auch als reale in Erscheinung tritt, wird die bisherige `Mittelstimme` frei für eine zusätzliche homorhythmische Begleitstimme. Dabei lässt sich die an op. 63,3 gemachte Beobachtung Charles Rosens, Chopins kontrapunktischer Stil sei »absolutely pianistic « ${ }^{28}$, auch auf op. 24,4 übertragen: So sind das chromatische `Zusammenziehen` der rechten Hand, das Herausschälen der Melodie aus der virtuellen Zweistimmigkeit sowie deren zusätzliche Anreicherung durch Wechselnoten und Appoggiaturen allesamt haptisch-taktil erfahrbare Vorgänge, die gleichsam wie aus der Klaviatur sertastets scheinen. Kontrapunkt und akkordische Begleitstruktur stehen zwischen Improvisation und Komposition. Im Verwischen dieser Grenze liegt ein besonderer Reiz der Mazurken, denen, obgleich höchst konstruiert, ein Charakter von Unmittelbarkeit und Flüchtigkeit eigen ist. ${ }^{29}$

In der Coda ab Takt 115 entspannt sich über einem ausgedehnten tonikalen Orgelpunkt eine neue Melodie, die aus der andersartigen Fortführung der >Mittelstimmeく Takt $6 \mathrm{ff}$. entwickelt ist. Einer wirklichen Entfaltung des Materials aber stehen der im fünften und sechsten Takt der Phrase verfrüht vollzogene Schluss und dessen geringfügig modifizierte Wiederholung entgegen, die die reguläre Achttaktigkeit des Periodenbaus gewährleistet (T. 119-122). Bei der Wiederaufnahme der gesamten Taktgruppe (T. 127-130) bleibt die Oberstimme im sechsten Takt auf dem Schlusston liegen; zur Wiederholung der melodischen Schlusswendung kommt es (zunächst) nicht mehr; nur noch das Akkordpendel vollzieht die Kadenz. Ab Takt 131 tritt an die Stelle des vormaligen Achttakters ein Viertakter, der gleichwohl auf den vorigen Schluss zurückgreift. Diese Taktgruppe wird ebenfalls wiederholt (T. $135 \mathrm{ff}$.). Hier nun bricht die melodische Figur bereits nach dem dritten Takt ab. Die Begleitung verharrt zunächst auf dem übermäßigen Quintsextakkord und versinkt dann unter dem Melodieschlusston in harmonischen Terzfällen von $\mathrm{F}$ über $\mathrm{d}$ nach B. Auch die letzten Takte vermeiden die reguläre V-I-Kadenz. Ganz am Ende aber steht die aus den Wiederholungen gleichsam herausdestillierte melodische Schlusswendung: Unbegleitet verklingt sie im Einzelton, mit demselben isolierten $f^{1}$, mit dem das Stück begann. Die Mazurka erlischt so in der Einstimmigkeit.

27 Zu diesem Entwicklungs- und Steigerungsprinzip vgl. Kallberg 1988, 15.

28 Rosen 1995, 451. Vgl. auch Kinzler 1977.

29 Analog könnte man die Ursache für die häufig verwendeten Satzmodelle wie dem erwähnten chromatischen Fauxbourdon, dem Parallelismus oder auch der Mixturtechnik in der Nähe der Mazurken zur volksmusikalischen Improvisation sehen. Zur Bedeutung der Improvisation im 19. Jahrhundert vgl. Felbick 2005. 

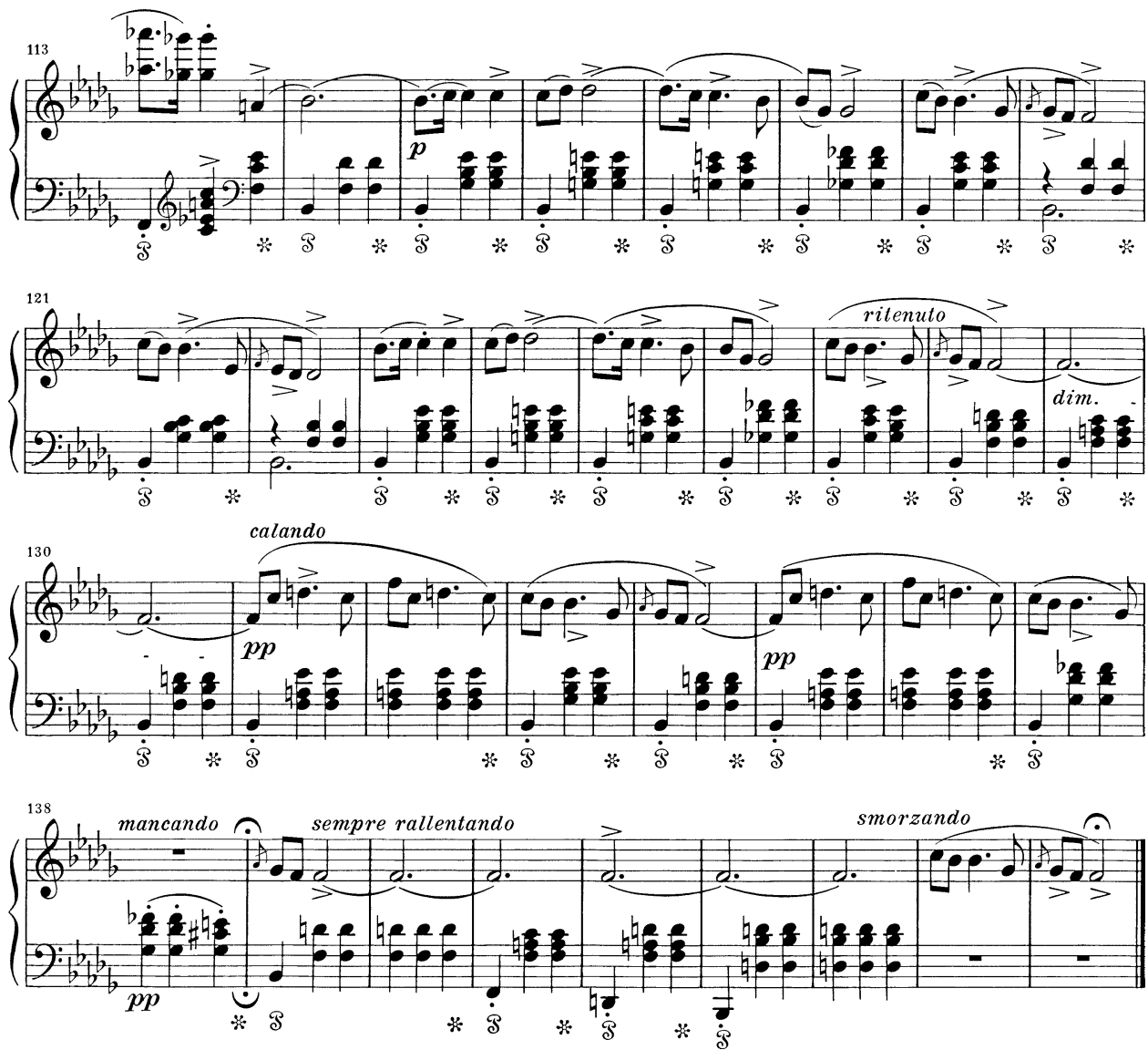

Beispiel 9: Frédéric Chopin, Mazurka op. 24,4, T. 113-146

Der lange Orgelpunkt lässt das Absinken des Basses in Terzen umso wirkungsvoller erscheinen. Hinzu tritt ein feinsinniges Spiel mit der metrischen Struktur. Durch die harmonische Verlängerung des übermäßigen Quintsextakkordes in Takt 138 - ein einkomponiertes rubato - erscheint der erwartete Vierer ${ }^{30}$ zu einem Fünfer gedehnt (T. 135-139). Takt 139 ist jedoch als Folge einer Phrasenverschränkung zugleich der Beginn des neuen Vierers. Regulär müsste das akzentuierte $f^{1}$ nicht in Takt 142, sondern einen Takt später erscheinen und mit Erreichen der Tonika zusammenfallen. Chopin zieht den Ton jedoch um einen Takt vor, so dass er mit dem Terzfall nach $d$ zusammenfällt. Unter strikter Wahrung der viertaktigen Periodik gestaltet er mit einer vieldeutigen Binnenstruktur die auf dem Wiederholungsprinzip beruhende Coda auch auf metrischer Ebene als einen unabgeschlossenen Vorgang.

30 Zum Thema »four-bar phrases« vgl. Rosen 1995, 258-278. 

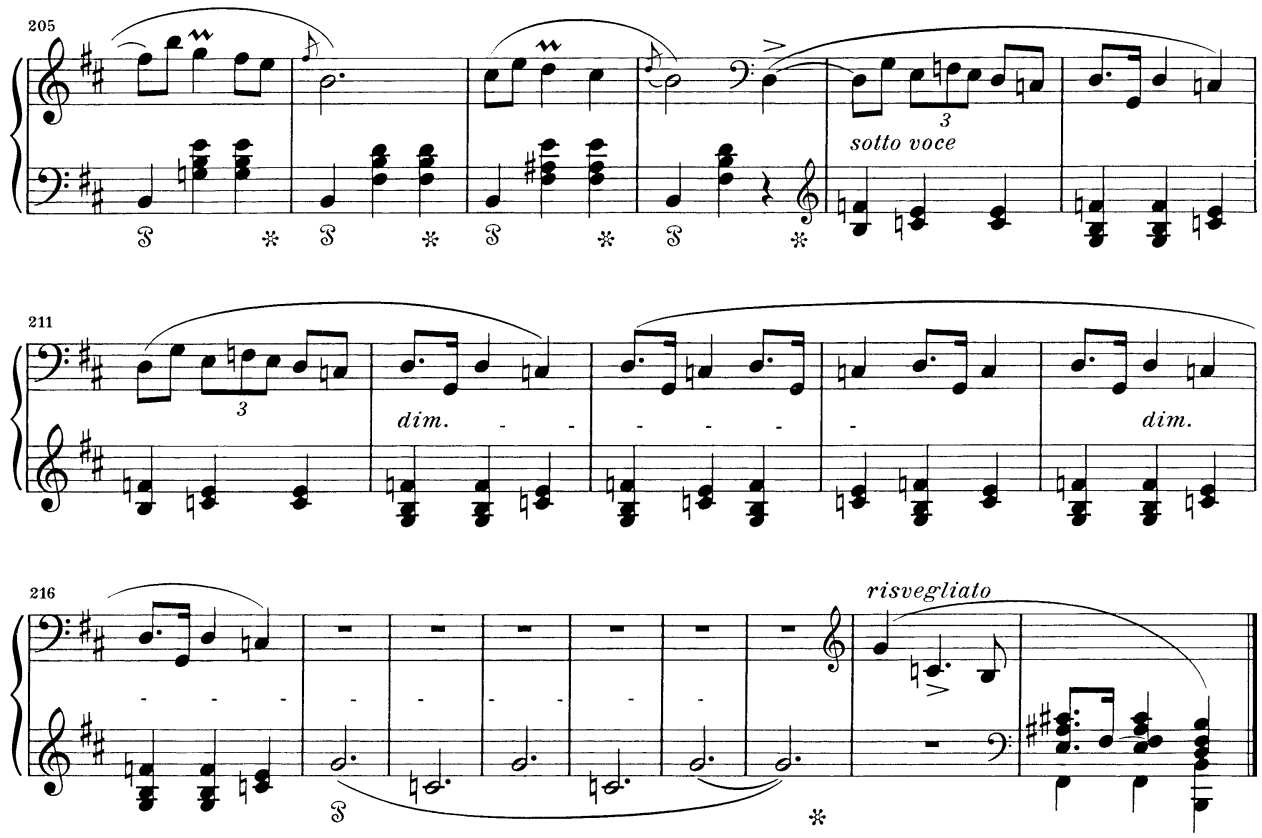

Beispiel 10: Frédéric Chopin, Mazurka op. 33,4, T. 205-224

\section{Alternative Enden - harmonische und metrische Charakteristika}

Eine Systematisierung der von Chopin angewendeten harmonischen und metrischen Verfahren kann an dieser Stelle nicht erfolgen. Auf zwei seiner Charakteristika soll dennoch abschließend eingegangen werden:

In der Mazurka op. 33,4 rivalisiert im A-Teil zunächst die neapolitanische Tonart C-Dur mit der Haupttonart h-Moll: Statt der erwarteten Korrespondenz von Halb- und Ganzschluss im periodisch gebauten Hauptthema kommt es am Ende des Nachsatzes zur Etablierung von C-Dur als eigener tonaler Ebene (T. $16 \mathrm{ff} . \mathrm{m}$. A. bzw. T. $41 \mathrm{ff} . \mathrm{m}$. A. infolge der ausnotierten Wiederholung des gesamten ersten Formteils). Dadurch dass die Fortsetzug des A-Teils in B-Dur steht (T. 49 ff.), wird die Haupttonart h-Moll von beiden Seiten halbtönig eingekreist. Das melodische Charakteristikum einer jeden neapolitanischen Kadenz die enge Einfassung des Grundtones wird so auf die tonale Disposition übertragen. Auf einen B-Teil in H-Dur (T. 129 ff.) folgt die stark verkürzte Reprise des ATeils. Sie gelangt nur bis zum erstmaligen Erreichen des Nachsatzes; die Wiederholung der modifizierten Periode sowie die Fortsetzung in B-Dur bleiben ausgespart. Stattdessen wird die C-Dur-Passage in beständiger Wiederholung des harmonischen Oberquintpendels von 6 auf 14 Takte erweitert: Die Musik scheint ihr tonales Zentrum vergessen zu haben und klingt mit der melodischen Folge $g^{1}-c^{1}-g^{1}-c^{1}-g^{1}$ in langen Notenwerten aus (T. 217-222). Im Sinne von Nicholas Temperley ließe sich von diesem sharmonischen 

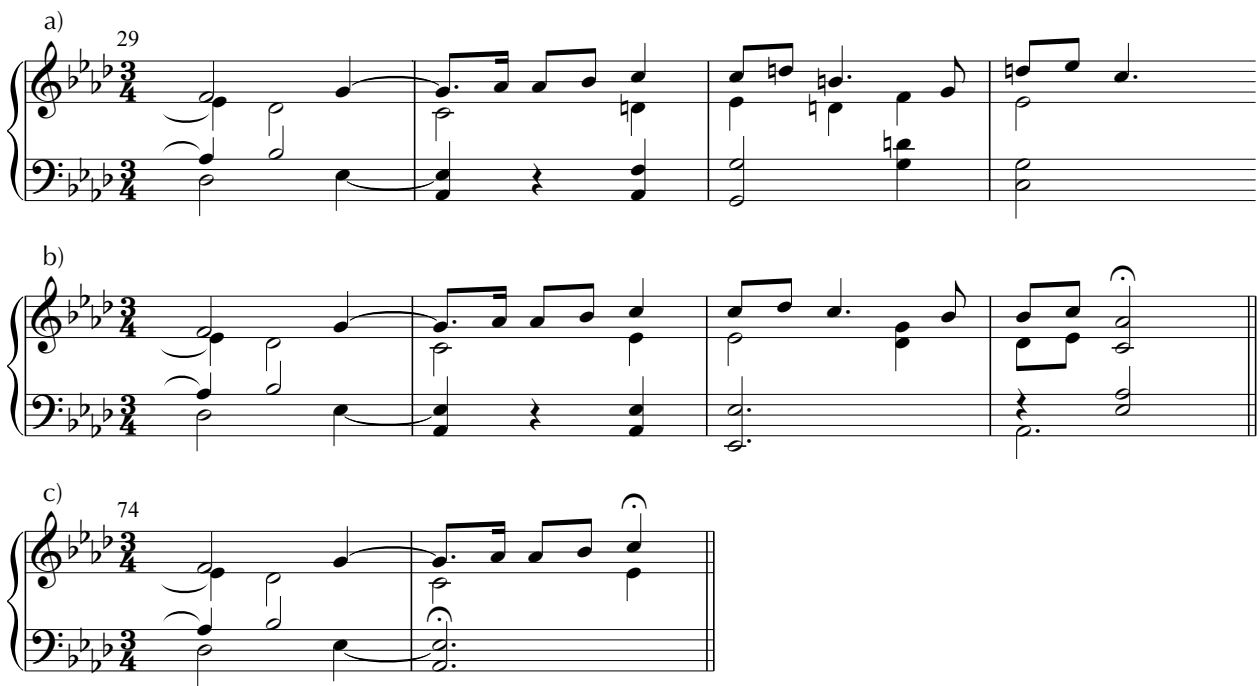

Beispiel 11: Frédéric Chopin, Mazurka op. 41,3; a) T. 29-32, b) mögliche Schlussbildung, c) tatsächlicher Schluss T. 71-74

Abdriften als einem »harmonic daydream « ${ }^{31}$ sprechen - ein kontemplativer Moment, den die beiden letzten Takte energisch «korrigieren`, indem sie abrupt nach h-Moll zurückführen (Beispiel 10).

Eine andere und ungleich radikalere Schlussgestaltung findet sich in op. 41,3: Die letzten 14 Takte des Stückes sind bereits bekannt, wenn sie am Ende der Komposition erscheinen; sie erklangen (mit Ausnahme der Harmonisierung des letzten Taktes) schon als Takte 17-30. Dort pendelt die Musik zwischen der Haupttonart As-Dur und c-Moll. Letzteres setzt sich schließlich durch (T. 31/32; Bsp. 11a) und wird im folgenden Teil zum variantem C-Dur aufgehellt. Diese Modulation ist freilich als Schluss ungeeignet, die letzten Takte müssen folglich nach As-Dur umgeschrieben werden (Bsp. 11b). Diese naheliegende Möglichkeit wählt Chopin nicht. Rein harmonisch betrachtet ist ein sinnvoller

31 Temperley 1987, 303. Bereits Schumann spricht in seinem fiktiven Bericht an Jeanquirit in Augsburg über den letzten kunsthistorischen Ball beim Reacteur den Aspekt des Traumhaften in Chopins Klavierspiel an und serzähltı darin, »daß es schon ein unvergessliches Bild gäbe, ihn [Chopin] wie einen träumerischen Seher am Klavier sitzen zu sehen, und wie man sich bei seinem Spiele wie der von ihm erschaffene Traum vorkäme, und wie er die heillose Gewohnheit habe, nach dem Schlusse jedes Stückes mit einem Finger über die pfeifende Claviatur hinzufahren, sich gleichsam mit Gewalt von seinem Traum loszumachen, und wie er sein zartes Leben schonen müsse [...].« In diesem 1837 für die Neue Zeitschrift für Musik verfassten Beitrag mag Schumann Eindrücke seiner persönlichen Begegnung mit Chopin in Leipzig vom 12. September des Vorjahres verarbeitet haben. Frappierend ist, dass sich seine Schilderung (»sich gleichsam mit Gewalt von seinem Traum loszumachen«) wie ein Vorgriff auf die Schlussgestaltung von dessen op. 33,4 ausnimmt - ein Werk das erst 1838 vorlag. (1914, Bd. 1, 258). Dass Chopin für die Schlußkadenz risvegliato (wieder erwacht) verlangt, bestärkt in der Triftigkeit dieser Assoziation. 
Schlussklang bereits in Takt 14 erreicht, der aber als zweiter Takt eines Vierers formal in der Luft hängt. ${ }^{32}$

Die Zuhörer müssen das Stück in der Hörvorstellung imaginär zu Ende führen, denn Chopin lässt die Passage bereits nach 14 statt nach 16 Takten unvermittelt -

\section{Literatur}

Borris, Siegfried (1963), „Chopins Bedeutung für den Chromatismus des 19. Jahrhunderts", in: The Book of the $1^{\text {st }}$ International Musicological Congress devoted to the works of Frédéric Chopin, hg. von Zofia Lissa, Warschau: PWN, Polish Scientific Publishers Warszawa, 107-110.

Chailley, Jacques (1963), „L'importance de Chopin dans l'évolution du langage harmonique", in: The Book of the $1^{\text {st }}$ International Musicological Congress devoted to the works of Frédéric Chopin, hg. von Zofia Lissa, Warschau: PWN, Polish Scientific Publishers Warszawa, 30-43.

Cone, Edward T. (1968), Musical Form and Musical Performance, New York: Norton.

Danuser, Hermann (1995), „Das Ende als Anfang. Ausblick einer Schlußfigur bei Joseph Haydn«, in: Studien zur Musikgeschichte. Eine Festschrift für Ludwig Finscher, hg. von Annegrit Laubenthal, Kassel: Bärenreiter, 818-827.

Felbick, Lutz (2005), „Vom Einfluss der Improvisation auf das mitteleuropäische Musikleben des 19. Jahrhunderts", Musiktheorie 20, 165-181.

Gerhard, Anselm (1996), »Reflexionen über den Beginn in der Musik. Eine neue Deutung von Chopins Préludes op. 28«, in: Deutsche Musik im Wegekreuz zwischen Polen und Frankreich. Zum Problem musikalischer Wechselbeziehungen im 19. und 20. Jahrhundert, hg. von Christoph Hellmut Mahling, Tutzing: Schneider 99-111.

Gołąb, Maciej (1995), Chopins Harmonik. Chromatik in ihrer Beziehung zur Tonalität, Köln: Bela.

Kallberg, Jeffrey (1988), »The problem of repetition and return in Chopin's mazurkas", Chopin Studies 1, hg. von Jim Samson, Cambridge: University Press, 1-25.

_ (1996) „Chopin's last style« in: ders., Chopin at the boundaries. Sex, history and musical genre, Cambridge (Mass.): Harvard University Press.

Kinzler, Hartmuth (1977), Frédéric Chopin, Über den Zusammenhang von Satztechnik und Klavierspiel (= Freiburger Schriften zur Musikwissenschaft 9), München und Salzburg: Katzbichler 1977.

Kurth, Ernst (1923), Romantische Harmonik und ihre Krise in Wagners 'Tristanı, 3. Aufl., Berlin: Max Hesse, Reprint Hildesheim u. a.: Olms 1985.

Lissa, Zofia (1973), „Klavierpolonaise und -mazurka im 19. Jahrhundert», in: Gattungen der Musik in Einzeldarstellungen. Gedenkschrift Leo Schrade, hg. von Wulf Arlt u.a., Bern und München: Francke, 813-839.

32 Die Mazurka wahrt - bis auf den Schluss - strikt die Ordnung in Vierern. 
Metzner, Edith (2004), »Chopins Mazurken«, Musiktheorie 19, 377-384.

Rosen, Charles (1995) The Romantic Generation, Cambridge (Mass.): Harvard University Press.

Schaefer, Janina (2007), Frédéric Chopin: Die Mazurken, Univ. Diss. München.

Schumann, Robert (1914), Gesammelte Schriften über Musik und Musiker, hg. von Martin Kreisig, 2 Bde., Leipzig: Breitkopf \& Härtel.

Stierle, Karlheinz und Rainer Warning (Hgg.) (1996), Das Ende. Figuren einer Denkform, München: Fink.

Temperley, Nicholas (1980), „Chopin, 9. Harmony«, in: The New Grove dictionary of music and musicians, hg. von Stanley Sadie, London: Macmillan, 302-304.

Torkewitz, Dieter (2004), »Eine unendliche Geschichte oder: Von der Emanzipation des Einzeltons, Musiktheorie 19, 177-187. 